\title{
LIPIDOS PLASMATICOS Y POLIMORFISMO Xbal DEL GEN DE LA APOB-100 EN UN GRUPO DE NIÑOS, Y JOVENES Y SUS PADRES
}

\section{PLASMA LIPIDS AND XbaI POLYMORPHISM ON APOB-100 GENE IN A GROUP OF CHILDREN AND THEIR PARENTS}

\author{
Nelsy Loango ${ }^{1}$, Beatriz Restrepo ${ }^{2}$, Ana Lucia Torres ${ }^{3}$, Patricia Landázuri ${ }^{4}$ \\ ${ }^{1}$ Grupo de Investigación en Enfermedades Cardiovasculares y Metabólicas. Programa de Biología. Facultad de Ciencias Básicas y Tecnologías. Universidad del \\ Quindío. \\ ${ }^{2}$ Grupo de Investigación en Enfermedades Cardiovasculares y Metabólicas. Programa de Medicina. Facultad Ciencias de la Salud. Universidad del Quindío. \\ ${ }^{3}$ Docente Investigadora Pontificia Universidad Javeriana \\ ${ }^{4}$ Grupo de Investigación en Enfermedades Cardiovasculares y Metabólicas. Programa de Medicina. Facultad Ciencias de la Salud. Universidad del Quindío.
}

Fecha de recibido: febrero 3 de 2010

Fecha Aceptado: Junio 9 de 2010-10-30

Correspondencia: Facultad Ciencias de la Salud. Universidad del Quindío, Av. Bolívar Calle 12 norte Armenia Quindío. Correo electrónico: plandazu@uniquindio.edu.co.

\section{RESUMEN}

Introducción: Algunos estudios ha mostrado asociación entre el polimorfismo Xbal del gen de la apolipoproteína $B$ (apoB) y niveles plasmáticos de lípidos y apoproteína $B$, pero otros no. Objetivo: determinar la relación entre lípidos plasmáticos, apo $B$ y el polimorfismo Xbal en un grupo de niños y jóvenes de ambos sexos entre 4 y 18 años y sus padres. Métodos: Se estudiaron 63 niños y 85 padres. Los niños se dividieron en dos grupos: niños con hipercolesterolemia (CHC), colesterol total (CT)>175 mg/dly niños sin hipercolesterolemia $(S H C), C T<175 \mathrm{mg} / \mathrm{dl}$. Se determinaron: genotipo Xbal (por la reacción en cadena de la polimerasa (PCR) y digestión con la enzima de restricción Xbal), perfil lipídico, apB y apoA1. Resultados: De los individuos estudiados tenían genotipo X1X1, 28,37\%, X1X2 64,86\%, y X2X2 6,75\%. La frecuencia alélica fue de 0,61 (X1) y 0,40 (X2). La frecuencia del genotipo X1X1 fue de 0,39 y 0,28 para niños CHC y SHC respectivamente y de 0,30 y 0,19 en sus padres respectivamente. No se encontraron diferencias en los niveles de CT y C-LDL, entre los alelos X1 y el X2 en cada grupo. En el grupo de padres e hijos CHC, el alelo X2 tuvo niveles de triglicéridos (TG), más altos, sin ser significativos. Conclusiones: Las frecuencias alélicas y génicas son similares a las descritas para poblaciones occidentales y diferentes a las reportadas para la población oriental. En esta población no se encontró relación entre el polimorfismo Xbal del gen de la apolipoproteína $B$ (apoB y los niveles de lípidos y $a p o B$, sin embargo, se necesitan estudios complementarios.

Palabras clave: apoproteína B, polimorfismo Xbal, perfil lipídico, niños, colesterol.

\begin{abstract}
Introduction: Some studies have shown association between Xbal polymorphism of apolipoprotein $B$ (apoB) gene and serum levels of lipids and apolipoprotein B, but not in others. Objective: to determine the relationship between plasma lipids, apoB, Xbal polymorphism in a group of boys and girls between 4 and 18 years and their parents. Methods: We studied 63 children and 85 parents. The children were divided into two groups: children with hypercholesterolemia (CHC), total cholesterol (TC)>175 $\mathrm{mg} / \mathrm{dl}$ and children without hypercholesterolemia $(\mathrm{SHC}), \mathrm{TC}<175 \mathrm{mg} / \mathrm{dl}$, were determined: Xbal genotype (for the polymerase chain reaction (PCR) and digestion with Xbal restriction enzyme), lipid profile, ApoB and apoA1. Results: Individuals were X1X1 genotype, $28.37 \%, 64.86 \% \times 1 X 2$, and $6.75 \% \times 2 X 2$. The allele frequency was 0.61 (X1) and 0.40 (X2). X1X1 genotype frequency was 0.39 and 0.28 children $\mathrm{CHC}$ and SHC respectively and 0.30 and 0.19 in parents respectively. There were no differences in total cholesterol and LDL-C, between X1 and X2 alleles in each group. In the group of parents and children CHC, the X2 allele showed levels of triglycerides (TG), higher, although not was significant. Conclusions: Allele frequencies and gene were similar to those described for Western populations and different from those reported for the eastern population. In this population, no relationship was found between the polymorphism and levels of lipids and apoB, however, additional studies are needed.
\end{abstract}

Key words: Apolipoprotein B, Xbal polymorphism, lipids profile, children, cholesterol. 


\section{INTRODUCCIÓN}

Elevadas concentraciones de apolipoproteína B (apoB) en sangre son consideradas un importante factor de riesgo para enfermedad coronaria.1 La apoB es el mayor ligando fisiológico para el receptor de las lipoproteínas de baja densidad (LDL) y por lo tanto juega un papel dominante en la homeostasis del colesterol2. La apoB existe en el plasma como dos isoformas apoB-48 (sintetizada en intestino) y apoB-100 (sintetizada en hígado; su gen está situado en el brazo corto del cromosoma 23. En el se han encontrado algunos polimorfismos de longitud de fragmentos de restricción, los cuales parecen modificar los niveles de lípidos y lipoproteínas plasmáticas en los individuos4,5. El polimorfismo de la enzima de restricción Xbal en el gen de la apoB-100, es uno de ellos y ocurre en la tercera posición del codón treonina 2488 en la región codificante del gen.4-6 Algunas publicaciones referidas a adultos demuestran asociaciones entre los distintos genotipos del polimorfismo Xbal y los niveles plasmáticos de colesterol total (CT) y colesterol en las LDL (C-LDL), 6 sin embargo, los estudios en población infantil son escasos 7,8 y con resultados variables dependiendo de la etnicidad, el sexo y factores ambientales. El objetivo de este trabajo fue estudiar la asociación entre los niveles de lípidos y lipoproteínas plasmáticas y el polimorfismo de Xbal en el gen de la apoB-100 en un grupo de niños y sus padres.

\section{MATERIALES Y MÉTODOS}

Población de Estudio: Se diseño un estudio corte transversal con muestra condicionada que incluía niños y jóvenes de ambos sexos con edades entre 4 y 18 años con hipercolesterolemia ( $\mathrm{CHC}$ ) y sin hipercolesterolemia (SHC). Los niños CHC se seleccionaron a partir de una muestra representativa de las historias clínicas pediátricas de los centros asistenciales y laboratorios clínicos públicos y privados de la Cuidad de Armenia, Departamento del Quindío en Colombia. Se incluyeron los niños en cuyas historias clínicas aparecía un reporte de colesterol total mayor de $175 \mathrm{mg} / \mathrm{dl}$ (4,5 mmol/L), valores definidos en varios programas sobre tratamiento de dislipidemias en niños.9,10 como de riesgo moderado de padecer enfermedades cardiovasculares (ECV). Los niños SHC se seleccionaron basados en un muestreo aleatorio de diferentes colegios públicos de la ciudad, cuyos niveles de colesterol una vez medidos en el laboratorio no fueron mayores de $175 \mathrm{mg} / \mathrm{dl}$. Se excluyeron de ambos grupos niños con tratamiento dietario y farmacológico para dislipidemias, o enfermedades como diabetes o hipotiroidismo o daño renal.
En el estudio se incluyeron los padres de ambos grupos independientemente de su perfil lipídico. El proyecto contó con la aprobación del comité de bioética de la Facultad Ciencias de la Salud de la Universidad del Quindío.

Tamaño de la muestra: De las 100 historias clínicas seleccionadas con datos de colesterol mayor de $175 \mathrm{mg} / \mathrm{dl}$, solo aceptaron participar en el estudio y acudieron a la toma de muestra 36 niños con sus padres, 5 no cumplieron los criterios de inclusión. Del grupo sin hipercolesterolemia después de la convocatoria en los colegios se presentaron 40, pero se descartaron 8 por las mismas razones anteriores. El total de individuos del estudio fue de 148 incluyendo padres e hijos.

Tras el consentimiento informado por escrito a padres e hijos se les tomó una muestra de sangre por venopunción, en tubo seco para las pruebas de perfil lípidico y en tubos con EDTA para las pruebas genéticas, después de 12 horas de ayuno.

El colesterol y los triglicéridos (TG) fueron medidos mediante método enzimático utilizando un estuche comercial). El colesterol en las lipoproteínas de alta densidad ( $\mathrm{C}-\mathrm{HDL}$ ) se determinó en el sobrenadante obtenido de la precipitación del colesterol VLDL y LDL con ácido fosfotúngstico y cloruro de magnesio, El colesterol LDL se calculó mediante la fórmula de FriedewaldFredrikson11. Se calcularon las relaciones CT/HDL y LDL/HDL, las cuales se ha demostrado son mejores indicadores de enfermedad coronaria que el CT o las LDL independientes 12 .

El ADN fue extraído de sangre completa, con un estuche comercial (Promega). El polimorfismo Xbal de la ApoB-100 se realizó por amplificación específica de los alelos usando la reacción en cadena de la polimerasa, brevemente: para un volumen final de 50 I, la mezcla de reacción incluyó: 200 ng de ADN, tampón de reacción 1x, MgCl2 2.5 mM, dNTPs 100 $\mathrm{mM}$, Taq polimerasa 0,5 UI (Gibco/BRL) y los cebadores GGAGACTATTCAGAAGCTAA y GAAGAGCCTGAAGACTGACT $10 \mathrm{M}$ (Gibco/BRL). Los 30 ciclos de amplificación se efectuaron en un termociclador (Perkin Elmer 2400), con los siguientes tiempos y temperaturas: un ciclo inicial de desnaturalización a $95^{\circ} \mathrm{C}$ por 5 minutos y 29 ciclos incluyendo cada uno, desnaturalización $95^{\circ} \mathrm{C}, 1$ minuto, hibridización $51^{\circ} \mathrm{C} 30$ segundos y extensión $72^{\circ} \mathrm{C} 1$ minuto. Se hizo un ciclo final de extensión a $72^{\circ} \mathrm{C}$ por $10 \mathrm{~min}$. $20 \mathrm{l}$ del producto amplificado fueron digeridos con $10 \mathrm{UI}$ de la enzima de restricción Xbal (Gibco/BRL); los fragmentos de restricción fueron separados por electroforesis en gel de 
agarosa al $2 \%$ y visualizados en un transiluminador de luz ultravioleta tras tinción del gel con bromuro de etidio al $0,025 \%$. Los genotipos se determinaron y clasificaron por la ausencia o presencia de los fragmentos de restricción así, X1X1 un fragmento de 709 pares de bases (pb); X1X2 tres fragmentos de 709, 436 y 274 pb, y X2X2 dos fragmentos de 436 y $274 \mathrm{pb}$.

\section{ANÁLISIS ESTADÍSTICO}

En los resultados las variables se expresan como la media \pm la desviación estándar (DE). Se utilizó el test t de estudent para comparaciones entre dos grupos. La estimación de las frecuencias se realizó mediante el método de contaje alelico. Para comparar las frecuencias alélicas y genotípicas de aplicó el test de Chi cuadrado (X2). Se calcularon los intervalos de confianza para el $95 \%$ en todos los casos estudiados considerando que existía significancia estadística cuando $p<0,05$. El genotipo de muy baja frecuencia fue asignado a otros de acuerdo con el método estimado simple de genes13

\section{RESULTADOS}

En este estudio se incluyeron 148 individuos, 63 correspondieron a niños y jóvenes entre 4 y 18 años y 85 a sus padres. La tabla 1 muestra las características generales de los participantes en el estudio. Los grupos de padres fueron similares entre $s i$, en cuanto a sexo $(p=0,91)$, índice de masa Corporal (IMC) $(p=0,43)$ y edad $(p=0,45)$. Entre los dos grupos de niños estudiados no hubo diferencias en la distribución de la edad, sexo e IMC ( $p=0,053,0,77$ y 0,46 respectivamente).

La concentración de lípidos plasmáticos de la población general se muestra en la tabla 2. Como era de esperarse en el grupo de niños CHC, el CT, C-LDL y las relaciones CT/HDL y
LDL/HDL, fueron significantemente más altas que en el grupo SHC. A pesar que los padres de los niños $\mathrm{CHC}$ no fueron previamente seleccionados por sus niveles de colesterol, se encontró que el CT, C-LDL y las relaciones $\mathrm{CT} / \mathrm{HDL}$ y LDL/HDL fueron significantemente más altas en ellos, que en los padres de los niños SHC. No se encontró correlación significativa entre los lípidos de hijos y sus padres (madre o padre, datos no mostrados). Con relación a la apoproteína B, padres y niños CHC tienen concentraciones significantemente más elevadas de esta proteína que los niños SHC. Los niveles de significancia de todos los datos se muestran en la tabla 2.

Para la apoA, las concentraciones fueron significantemente más reducidas en los grupos de padres de niños SHC que en los padres de niños CHC. Lo mismo ocurrió en los niños pero la diferencia no fue significativa. Los niveles de triglicéridos siempre fueron más altos en padres y niños CHC comparados con el otro grupo de padres e hijos, pero solo fue estadísticamente significante entre los niños. Interesantemente en ambos grupos tanto niños como padres presentaron niveles bajos C-HDL. Los niveles de significancia de todos los datos se muestran en la tabla 2.

Las diferencias en la expresión de lípidos plasmáticos y apoproteínas encontradas entre los dos grupos de estudio, no pudo ser explicada por efecto del sexo ( $p$ entre hombres y mujeres de 0,1, 0,06 y 0,2 para CT, C-LDL y ApoB respectivamente en los padres de niños $\mathrm{CHC}$ y $p=0,39,0,3$ y 0,39 en padres de niños SHC para las mismas variables). Similar situación se encontró en niñas y niños $\mathrm{CHC}$, siendo $\mathrm{p}$ $=0,42,0,45$ y 0,28 para CT, C-LDL y ApoB respectivamente. En los niños SHC a pesar de no encontrar diferencias en la edad, las niñas presentaron niveles de colesterol y C-LDL más altos que los niños ( $p=0,02$ y 0,002 respectivamente), no encontramos diferencia por sexo en la concentración de apoB. Tampoco la edad pudo explicar las diferencias

Tabla 1. Comparación sexo, edad e índice de masa corporal para los grupos de estudio.

\begin{tabular}{|c|c|c|c|c|c|c|}
\hline \multirow[b]{2}{*}{ Variable } & \multicolumn{3}{|c|}{ Padres } & \multicolumn{3}{|c|}{ Hijos } \\
\hline & $\mathrm{CHC}$ & $\mathrm{SHC}$ & $P$ & $\mathrm{CHC}$ & $\mathrm{SHC}$ & $p$ \\
\hline $\mathrm{n}$ & 43 & 42 & & 31 & 32 & \\
\hline Sexo M/F & $21 / 22$ & $20 / 22$ & & $16 / 15$ & $16 / 16$ & \\
\hline Edad & $41,0 \pm 9,1$ & $40,8 \pm 9,5$ & 0,45 & $12,5 \pm 3,8$ & $10,8 \pm 4,0$ & 0,053 \\
\hline IMC & $25,9 \pm 4,2$ & $25,8 \pm 4,1$ & 0,43 & $19,5 \pm 3,8$ & $19,6 \pm 3.2$ & 0,46 \\
\hline $\mathrm{n}$ total & 85 & & & 63 & & \\
\hline
\end{tabular}

$\mathrm{CHC}=$ con hipercolesterolemia. $\mathrm{SHC}=$ sin hipercolesterolemia 
encontradas entre los grupos, siendo los coeficientes de correlación entre edad, CT, C-LDL y ApoB $<0,5$ para todos los grupos (datos no mostrados).

Tabla 2. Distribución de lípidos sanguíneos en la población general.

\begin{tabular}{|c|c|c|c|c|c|c|}
\hline \multirow[b]{2}{*}{ Variable } & \multicolumn{3}{|c|}{ Padres } & \multicolumn{3}{|c|}{ Hijos } \\
\hline & $\mathrm{CHC}$ & $\mathrm{SHC}$ & $\mathrm{P}$ & $\mathrm{CHC}$ & $\mathrm{SHC}$ & $\mathrm{P}$ \\
\hline $\mathrm{n}$ & 43 & 42 & & 31 & 32 & \\
\hline $\mathrm{CT}, \mathrm{mmol} / \mathrm{L}$ & $5,7 \pm 1,4$ & $4,7 \pm 1,1$ & 0,0002 & $5,6 \pm 1,0$ & $3,7 \pm 0,5$ & $<0,0001$ \\
\hline $\mathrm{HDL}, \mathrm{mmol} / \mathrm{L}$ & $1,0 \pm 0,3$ & $1,05 \pm 0,3$ & 0,50 & $1,2 \pm 0,5$ & $1,1 \pm 0,3$ & 0,24 \\
\hline $\mathrm{TG}, \mathrm{mmol} / \mathrm{L}$ & $1,9 \pm 1,1$ & $1,6 \pm 1,2$ & 0,13 & $1,6 \pm 1,9$ & $0,9 \pm 0,5$ & 0,026 \\
\hline $\mathrm{LDL}, \mathrm{mmo} / \mathrm{L}$ & $4,3 \pm 1,3$ & $3,4 \pm 1,1$ & 0,0003 & $4,1 \pm 1,0$ & $2,4 \pm 0,5$ & $<0,0001$ \\
\hline ApoB mg/dl & $107,5 \pm 39,1$ & $88,2 \pm 32$ & 0,007 & $95,0 \pm 15,9$ & $70,0 \pm 14,6$ & $<0,0001$ \\
\hline ApoA mg/dl & $127,5 \pm 45,7$ & $111,9 \pm 44$ & 0,049 & $132,5 \pm 41,4$ & $130,6 \pm 41,8$ & 0,42 \\
\hline $\mathrm{CT} / \mathrm{HDL}$ & $5,7 \pm 1,7$ & $4,8 \pm 1,5$ & 0,005 & $5,7 \pm 4,7$ & $3,4 \pm 1,1$ & 0,006 \\
\hline LDL/HDL & $4,3 \pm 1,5$ & $3,4 \pm 1,1$ & 0,0026 & $2,7 \pm 1,3$ & $2,2 \pm 0,9$ & 0,051 \\
\hline
\end{tabular}

$\mathrm{CHC}=$ con hipercolesterolemia. $\mathrm{SHC}=\sin$ hipercolesterolemia

La tabla 3 muestra la distribución genotípica y alélica de la población general y de los grupos. De los 148 individuos estudiados para este polimorfismo, 42 de ellos (28,37\%), tenían genotipo X1X1, 96 (64,86\%), presentaron genotipo $\mathrm{X} 1 \mathrm{X} 2$ y solo $10(6,75 \%)$ presentaron genotipo $\mathrm{X} 2 \mathrm{X} 2$. La distribución de frecuencias en la población estuvo de acuerdo a lo esperado para la ley del equilibrio de Hardy-
Weinbberg. Comparando los grupos $\mathrm{CHC}$ y los $\mathrm{SHC}$ en padres e hijos se encontró que la frecuencia del genotipo X1X1 fue más alta en los $\mathrm{CHC}$ comparados con los $\mathrm{SHC}$ tanto en padres $(0,30$ vs. 0,19$)$ como en hijos $(0,39$ vs 0,28$)$ respectivamente. Sin embargo esta diferencia no fue estadísticamente significativa ( $p>0,05$ en los dos grupos generacionales).

Tabla 3. Genotipos, frecuencias genotípicas y alélicas de la población de estudio

\begin{tabular}{|c|c|c|c|c|c|c|c|c|c|c|c|}
\hline \multicolumn{2}{|c|}{ Grupos } & \multicolumn{6}{|c|}{ Frecuencia genotípica } & \multicolumn{4}{|c|}{ frecuencia alélica } \\
\hline & & $\mathrm{X} 1 \mathrm{X} 1$ & & $\mathrm{X} 1 \mathrm{X} 2$ & & $\times 2 \times 2$ & & $\mathrm{X} 1$ & & $\mathrm{X} 2$ & \\
\hline & $\mathrm{CHC}$ & & 13 & & 27 & & 3 & & 53 & & 33 \\
\hline \multirow{4}{*}{ Padres } & $n=43$ & $(0,30)$ & & $(0,63)$ & & (007) & & $(0,61)$ & & $(0,39)$ & \\
\hline & $\mathrm{SHC}$ & & 8 & & 31 & & 3 & & 47 & & 37 \\
\hline & $n=42$ & $(0,19)$ & & $(0,73)$ & & $(0,07)$ & & $(0,55)$ & & $(0,44)$ & \\
\hline & & & 21 & & 58 & & 6 & & 100 & & 70 \\
\hline \multirow[t]{2}{*}{ Total padres } & $n=85$ & $(0,12)$ & & $(0,34)$ & & $(0,03)$ & & $(0,59)$ & & $(0,41)$ & \\
\hline & $\mathrm{CHC}$ & & 12 & & 16 & & 3 & & 40 & & 22 \\
\hline \multirow[t]{3}{*}{ Hijos } & $n=31$ & $(0,39)$ & & $(0,51)$ & & $(0,1)$ & & $(0,64)$ & & $(0,35)$ & \\
\hline & $\mathrm{SHC}$ & & 9 & & 22 & & 1 & & 40 & & 24 \\
\hline & $n=32$ & $(0,28)$ & & $(0,68)$ & & $(0,03)$ & & $(0,62)$ & & $(0,37)$ & \\
\hline \multirow[t]{2}{*}{ Total hijos } & $n=63$ & & 21 & & 38 & & 4 & & 80 & & 46 \\
\hline & & $(0,33)$ & & $(0,66)$ & & $(0,06)$ & & $(0,63)$ & & $(0,36)$ & \\
\hline \multirow[t]{2}{*}{ Total Población } & & & 42 & & 96 & & 10 & & 180 & & 116 \\
\hline & $n=148$ & $(0,28)$ & & $(0,64)$ & & $(0,06)$ & & $(0,61)$ & & $(0,39)$ & \\
\hline
\end{tabular}

$\mathrm{CHC}=$ con hipercolesterolemia. $\mathrm{SHC}=$ sin hipercolesterolemia

Debido a su baja frecuencia los individuos con genotipo $\mathrm{X} 2 \mathrm{X} 2$, ( 3 individuos en el grupo de padres $\mathrm{CHC}$, 3 individuos en el grupo SHC, 3 en el grupo de niños $\mathrm{CHC}$ y 4 individuos en el grupo de niños SHC), se incluyeron con los individuos
X1X2 en todos los análisis subsiguientes. Con respecto al género no se encontraron interacciones significativas entre esta variable y los genotipos descritos, el género no fue tomado en cuenta en los análisis subsiguientes. 
Loango,N; Restrepo,B; Torres, A.L; Landázuri,P. - 183 -

Tabla 4. Contribución alélica al fenotipo lipídico en padres

\begin{tabular}{|c|c|c|c|c|c|c|}
\hline \multirow{2}{*}{$\begin{array}{l}\text { Grupos } \\
\text { Variables }\end{array}$} & \multicolumn{3}{|c|}{$\mathrm{CHC}$} & \multicolumn{3}{|c|}{$\mathrm{SHC}$} \\
\hline & $\mathrm{X} 1$ & $\times 2$ & $P$ & $\mathrm{X} 1$ & $\mathrm{X} 2$ & $P$ \\
\hline Edad (años) & $40,8 \pm 9,8$ & $41,2 \pm 8,9$ & 0,46 & $43,1 \pm 8,5$ & $40,3 \pm 9,8$ & 0,02 \\
\hline $\mathrm{CT}(\mathrm{mmol} / \mathrm{L})$ & $5,7 \pm 1,4$ & $5,7 \pm 1,4$ & 0,47 & $4,8 \pm 1,4$ & $4,7 \pm 1,1$ & 0,07 \\
\hline $\mathrm{HDL}(\mathrm{mmol} / \mathrm{L})$ & $1,0 \pm 0,2$ & $1,01 \pm 0,3$ & 0,23 & $1,2 \pm 0,3$ & $1,0 \pm 0,2$ & 0,10 \\
\hline $\mathrm{LDL}(\mathrm{mmol} / \mathrm{L})$ & $4,4 \pm 1,3$ & $4,3 \pm 1,3$ & 0,40 & $3,3 \pm 1,4$ & $3,4 \pm 1,0$ & 0,40 \\
\hline TG (mmol/L) & $1,6 \pm 0,7$ & $2,0 \pm 1,1$ & 0,10 & $1,6 \pm 1,3$ & $1,6 \pm 1,2$ & 0,40 \\
\hline IMC & $25,5 \pm 3,9$ & $26,1 \pm 4,4$ & 0,30 & $24 \pm 3,7$ & $26,2 \pm 4,1$ & 0,08 \\
\hline $\mathrm{IA}$ & $5,8 \pm 1,7$ & $5,7 \pm 1,8$ & 0,40 & $4,3 \pm 1,6$ & $4,9 \pm 1,5$ & 0,10 \\
\hline LDL/HDL & $4,5 \pm 1,5$ & $4,3 \pm 1,5$ & 0,30 & $3,0 \pm 1,4$ & $3,5 \pm 1,4$ & 0,16 \\
\hline APOB & $106,4 \pm 31,2$ & $108,0 \pm 42,6$ & 0,43 & $88,2 \pm 37,2$ & $88,3 \pm 31,7$ & 0,08 \\
\hline APO A & $126,4 \pm 40,9$ & $128,8 \pm 47,8$ & 0,43 & $120,5 \pm 46,6$ & $109 \pm 43,9$ & 0,28 \\
\hline
\end{tabular}

$\mathrm{CHC}=$ con hipercolesterolemia. $\mathrm{SHC}=$ sin hipercolesterolemia

Dentro de cada grupo de estudio (intragrupo) se examinó la contribución alélica a la expresión de los lípidos plasmáticos (tablas 4 y 5). Al comparar el alelo X1 con el X2 en cada grupo, no se encontraron diferencias significativas en los niveles de CT, sin embargo, los datos muestran una tendencia a ser más alto este lípido en los $X 1$ que en los $X 2$ en los padres SHC $(4,8 \pm 1,4$ y $4,7 \pm 1,1 p=0,07$ respectivamente) $y$ en los dos grupos de niños $(5,9 \pm 1,35,4 \pm 0,8$ para $\mathrm{CHC} y$ $3,8 \pm 0,4$ y $3,7 \pm 0,6$ para los $\mathrm{SHC}$ ).

La misma tendencia se encontró para el C-LDL donde los niveles fueron más altos para los $\mathrm{X} 1$, que para los $\mathrm{X} 2$, excepto para los padres SHC, aunque de nuevo, las diferencias no fueron significativas. En sentido inverso los niveles de TG, fueron más altos en el alelo X2 de los padres e hijos de CHC, pero no en los dos grupos SHC, sin ser la diferencia significativa. Igual situación se presentó con el IMC siempre más alto en los X1 que en los X2 en padres; en hijos solo fue más alto en los SHC sin que existan diferencias significativas en ningún grupo. Las apoB fueron mayores en los grupos X1 que en los X2 en los niños pero tampoco se encontraron diferencias significativas.

Tabla 5. Contribución alélica al fenotipo lipídico en niños

\begin{tabular}{lrrrrrr}
\hline Grupo & \multicolumn{3}{c}{ CHC } & \multicolumn{3}{c}{ SHC } \\
\cline { 2 - 7 } & \multicolumn{1}{c}{ X1 } & \multicolumn{1}{c}{$\mathrm{X}$} & \multicolumn{1}{c}{ X1 } & \multicolumn{1}{c}{ X } & $\mathrm{P}$ \\
\hline Edad (años) & $9,4 \pm 2,7$ & $11,74 \pm 4,6$ & 0,04 & $13,11 \pm 3,5$ & $12,2 \pm 4,0$ & 0,27 \\
CT(mmol/L) & $5,9 \pm 1,3$ & $5,4 \pm 0,8$ & 0,13 & $3,8 \pm 0,4$ & $3,7 \pm 0,6$ & 0,25 \\
HDL(mmol/L) & $1,2 \pm 0,5$ & $1,2 \pm 0,4$ & 0,49 & $1,1 \pm 0,3$ & $1,2 \pm 0,52$ & 0,41 \\
LDL(mmol/L) & $4,4 \pm 1,4$ & $3,8 \pm 0,7$ & 0,08 & $2,4 \pm 0,4$ & $2,3 \pm 0,5$ & 0,25 \\
TG (mmol/L) & $1,2 \pm 0,9$ & $1,9 \pm 2,4$ & 0,10 & $1,0 \pm 0,6$ & $0,85 \pm 0,52$ & 0,22 \\
IMC & $18,1 \pm 4,4$ & $20,4 \pm 3,3$ & 0,60 & $20,3 \pm 2,5$ & $19,3 \pm 3,5$ & 0,14 \\
IA & $6,6 \pm 6,5$ & $5,1 \pm 3,2$ & 0,24 & $3,4 \pm 0,9$ & $3,4 \pm 1,2$ & 0,44 \\
LDL/HDL & $3,0 \pm 1,6$ & $2,6 \pm 1,2$ & 0,20 & $2,3 \pm 0,7$ & $2,3 \pm 1,0$ & 0,40 \\
APOB & $95,5 \pm 18,5$ & $94,7 \pm 14,6$ & 0,44 & $70,8 \pm 10,5$ & $69,7 \pm 16,1$ & 0,40 \\
APO A & $135,6 \pm 42,8$ & $130,5 \pm 41,6$ & 0,37 & $119,1 \pm 36,0$ & $135,0 \pm 43,9$ & 0,15 \\
\hline
\end{tabular}

$\mathrm{CHC}=$ con hipercolesterolemia. $\mathrm{SHC}=$ sin hipercolesterolemia

\section{DISCUSIÓN}

La asociación de los polimorfismos más comunes en genes candidatos a que tienen una aparente influencia sobre las concentraciones plasmáticas de los lípidos y las lipoproteínas ha sido estudiada casi exclusivamente en adultos, en quienes la manifestación de dislipidemias es frecuente, sin embargo, tal como lo muestran estos resultados, la dislipidemia puede manifestarse desde edades tempranas como en el grupo de niños estudiados. Es necesario indicar que en el intervalo de edad estudiado (4-
18 años), se incluye la etapa puberal, con factores exógenos y endógenos propios de la edad, incluyendo cambios psicosociales y dietarios que pueden influenciar marcadamente el metabolismo lipídico y así complicar el establecimiento de cualquier asociación, en este estudio el efecto de la etapa puberal parece ser mínimo cuando se comparan y analizan sexo, edad e índice de masa corporal entre los grupos de niños sin hallar diferencias significativas.

Este trabajo muestra niveles de CT, LDL, TG y apoB más altos en los $\mathrm{CHC}$ y en sus padres, sin haber sido estos

Rev. Invest. Univ. Quindío (20): 179 - 186. Armenia - Colombia 
seleccionados por el perfil lipídico. Debido a ello, este grupo presentó una relación $\mathrm{CT} / \mathrm{HDL}$ y LDL/HDL más alta que I grupo de padres y niños sanos, lo que implica para los primeros mayor riesgo de sufrir enfermedades cardiovasculares. En el mismo sentido la alta concentración de triglicéridos encontrada en el grupo de padres e hijos CHC, se les comparó con padres e hijos SHC, constituye un problema potencial de salud cardiovascular para ellos. A este respecto, los resultados descritos aquí son similares a los presentados en otros estudios con niños,14,15 de otras región colombianas, y en grupo más amplio de niños quindianos16, donde registran una alta prevalencia de varios factores de riesgo en la población estudiada.

Sobre la base de la evidencia obtenida de muchos años de estudios experimentales y epidemiológicos, los niveles altos de CT y C-LDL y bajos de C-HDL, se reconocen como factores de riesgo para las ECV, sin embargo nueva información, muestra la importancia de los niveles de apoB y apoA como predictores de riesgo1,17. Una razón por la cual la apoB puede ser un fuerte predictor de riesgo más que el C-LDL es que esta apoproteína está presente no solo en las LDL sino también en las VLDL, IDL (lipoproteínas de densidad intermedia) y en la lipoproteína a (Lp (a)), por lo tanto, la concentración plasmática de apoB, mide la presencia de la apoproteína en todas estas partículas consideradas aterogénicas y puede ser mejor predictor de riesgo que el CT o el C-LDL solamente. En este trabajo padres e hijos del grupo $\mathrm{CHC}$, presentaron niveles altos de $\mathrm{ApoB}$, lo que representa un factor de riesgo adicional a sus niveles de lípidos plasmáticos.

Las alteraciones en los lípidos de los padres de los niños $\mathrm{CHC}$ cuando se les compara con los padres de niños SHC, sin haber sido seleccionados por sus niveles de colesterol, como sus hijos, sugiere, aunque la herramienta estadística no lo confirma, que hay una asociación entre los lípidos de los padres y los lípidos en los hijos; varios estudios ya han demostrado esta relación18.

En adición a la variación cuantitativa en los niveles plasmáticos de apoB, descritos aquí, se conoce que la variación genética en el locus su gen puede ser un factor de riesgo independiente para ECV.5-7,19,20 De tal forma que esta investigación aporta datos al respecto, así, en relación con las frecuencias alélicas encontradas en la población de este estudio, la frecuencia X1 $(0,61)$ fue más baja que la X2 $(0,39)$, cuando se les compara con frecuencias encontradas en la población japonesa $(X 1=0,97$ y X2=0,033) promedio para dos estudios revisados en Zaman y colaboradores21), o con la población China (promedio de dos estudios revisados $\mathrm{X} 1=0,93$ y X2 0,07 en la misma publicación21). Las poblaciones asiáticas se han considerado con menor riesgo de ECV. Al comparar la frecuencia X1 del presente estudio, con la frecuencia $X 1( \pm 0,54)$, de varias poblaciones europeas y americanas blancas, se encontró que fue un poco más alta, mientras que la frecuencia X2 $(0,39)$, fue más baja que la X2 $( \pm 0,46)$ europea y americana26. Interesantemente las frecuencias encontradas en este estudio son muy similares a las registradas en una población del norte de Italia (X1=0,65 y X2=0,34), donde además, la incidencia de ECV es alta22; Por lo tanto, los datos muestran que la distribución alélica de la población incluida en este trabajo es más parecida a la población occidental, que a la oriental y muy similar a la italiana.

En cuanto a la asociación del fenotipo lipídico con los genotipos de la apoB, nuestros datos muestran en el grupo de padres e hijos $\mathrm{CHC}$, una tendencia del genotipo X1X1 a tener niveles más altos de CT, C-LDL y ApoB y mayores las relaciones $\mathrm{CT} / \mathrm{HDL}$ y $\mathrm{LDL} / \mathrm{HDL}$, cuando se les comparó con genotipo $X 1 X 2(X 1 X 2+X 2 X 2)$, en el mismo grupo sin ser significativa la diferencia. Por el contrario, los TG parecen ser influenciados por el genotipo X1X2. Al respecto y en relación al genotipo X1X1 y su influencia en los lípidos plasmáticos, los resultados de diversos estudios son contradictorios, Puri y colaboradores23, comunican que en pacientes con enfermedad coronaria el genotipo X1X1 fue más frecuente en aquellos que tenían altos niveles de apoB y VLDL, pero fue menos frecuente en los pacientes con CT alto, por el contrario, Guzmán y colaboradores en un estudio en mujeres brasileñas con ECV20, asocia X1X1 con altos niveles de CT y LDL. Por otra parte, Cavalli y colaboradores5, en una investigación con hombres hipercolesterolémicos, también de origen brasileño, no encontró asociación entre los tres genotipos y los niveles de lípidos. En cuanto al genotipo $\mathrm{X} 1 \mathrm{X} 2$, en un trabajo hecho por Ye y colaboradores24, en pacientes chinos con EC y sus controles se describió que este genotipo era más frecuente en los pacientes que en los controles, mientras el alelo X2, estaba asociado con bajos niveles de HDL y apoA, no encontró asociación con el CT. Por otro lado, Aalto describió para el mismo alelo X2, niveles altos de CT y LDL en población Finlandés25. Estos resultados contradictorios en diferentes poblaciones podrían atribuirse entre muchas causas, a diferencias raciales.

En relación con estas diferencias, la población de esta investigación, se parece más a los grupos de pacientes de Guzmán y colaboradores20, en cuanto a la asociación lipídica con los alelos X1 y X2, y difiere del estudio de Aalto25 en población Finlandés y de Ye24 en población china. En Colombia hasta donde se ha investigado no se conocen estudios que asocien este polimorfismo con los lípidos plasmáticos, por lo tanto, no se puede argumentar, si la 
tendencia observada en este trabajo es propia de nuestra heterogeneidad étnica, o similar a la comunicada por Guzmán20 para una población brasileña, o es una desviación debido al pequeño tamaño de la muestra. Los resultados indican que es necesario ampliar el estudio y sobre todo ver si el comportamiento es similar en otras regiones del país, con menos mezclas raciales, por ejemplo, en la población negra, para poder dar un significado y utilidad clínica a estos hallazgos.

\section{CONCLUSIONES}

Se concluye de este estudio que la población de niños y jóvenes $\mathrm{CHC}$ y sus padres tienen riesgo de sufrir ECV, por sus altos niveles de lípidos y apoproteínas. Las frecuencias alélicas y génicas encontradas son similares a las descritas para poblaciones occidentales y diferentes a las reportadas para la población oriental. En nuestra población contrario de lo que ha sido descrito en otras poblaciones el alelo X1 parece influenciar los niveles de CT, C-LDL y apoB y el alelo X2 parece influenciar los TG sin ser estadísticamente significativo este hallazgo. La relevancia clínica del polimorfismo Xbal no parece clara; para enfermedades multifactoriales como las cardiovasculares, la variación genética en el gen de la apoB estudiada independientemente, podría no tener un efecto directo sobre el perfil lipídico, esto enfatiza la importancia de realizar estudios como este, los cuales asociados a otros pueden ayudar en el futuro a la caracterización del perfil de riesgo cardiovascular de los individuos y poblaciones.

\section{BIBLIOGRAFÍA}

1. Olofsson SO, Bore'n J. Apolipoprotein B: a clinically important apolipoprotein which assembles atherogenic lipoproteins and promotes the development of atherosclerosis. J. Intern. Med. 2005;258: 395-410.

2. Smolenaars MM W, Madsen O, Rodenburg KW,. Van der Horst DJ. Molecular diversity and evolution of the large lipid transfer protein superfamily. J. Lipid Res. 2007. 48: 489-502.

3. Blackhart BD, Ludwing EM, Pierotti VR, Caiati L, Onasch MA, Wallis SC, Powell L, PeaseR, Knott TJ, Chu ML, Mahley RW, Scott J, MaCartthy BJ, Levy-Wilson B. et al. Structure of the human apolipoprotein B gene J. Biol Chem 1986; 261: 1536415367.

4. Real JT, Chaves FJ, Ejarque I, Garcia-Garcia AB, Valldecabres C, Ascaso JF, Armengod ME, Carmena R. Major apolipoprotein B-100 mutations in lipoprotein metabolism and atherosclerosis. Physiol Res. 2001; 50:337-43.

5. Cavalli SA, Hirata MH, Salazar LA, Diament J, Forti N, Giannini SD, et al. Apolipoprotein B gene polymorphism: prevalence and impact on serum lipid concentrations in hypercholesterolemic individuals from Brazil. Clin Chim Acta 2000; 302: 189-203

6. Boekholdt SM, Peters RJ, Fountoulaki K, Kastelein JJ, Sijbrands EJ. Molecular variation at the apolipoprotein B gene locus in relation to lipids and cardiovascular disease: a systematic meta-analysis. Hum Genet. 2003; 113(5):417-25.

7. Aalto-Setala K, Viikari J, Akerblom HK, Kuusela V, Kontula K. DNA polymorphisms of the apolipoprotein B and A4C-III genes are associated with variations of serum low density lipoprotein cholesterol level in childhood J. Lipid Res. 1991; 32: 1477-1487.

8. Hubacek JA, Pistulkova H, Pisa Z, Valenta Z, Skodova Z, Poledne R. Lack of an association between apolipoprotein B Xbal polymorphism and blood lipid parameters in childhood. Physiol Res. 1998; 47(2):89-93.

9. US Preventive Services Task Force. Screening for lipid disorders in children: US Preventive Task Force recommendation statement. Pediatrics. 2007;120(1). Available at: www.pediatrics.org/cgi/content/full/120/1/e215

10. Conferencia Consenso Lipidos en Pediatría. An Esp Ped 1998; Sup 118:1-8

11. Friedewald WT, Levy RI, Fredickson DS. Estimation of the concentration of low-density lipoprotein cholesterol in plasma without use of the preparative ultracentrifuge. Clin-chim. 1972; 18:499-502.

12. Kinosian B, Glick H, Garland G. Cholesterol and coronary heart disease: predicting risk by levels and ratios. Ann Intern Med. 1994; 121:641-647

13. Wilson PW, Myers RH, Larson MG, Ordovas JM, Wolf PA, Schaefer EJ. Apolipoprotein E alleles, dislipidaemia, and coronary heart disease: the Framingham Offspring study. JAMA 1994; 272:1666-1671.

14. Uscátegui RM, Alvarez MC, Laguado I, Soler W, Martinez I, Arias R, Duque B, Perez J, Camacho JA. Factores de Riesgo Cardiovascular en niños de 6-18 años en Medellín (Colombia) An Pediatr 2003; 58:411-417.

15. Poveda E, Callas N, Baracaldo C, Castillo C, Hernández P, Guerra M. Evaluación de las concentraciones de lípidos y apoproteínas A-I y B-100 en un grupo de escolares de cinco departamentos del centro-oriente de Colombia. Biomédica 2007;27:385-99. 
16. Landázuri P, Loango N, Gallego ML, Restrepo B. Diferencias de sexo, edad y lípidos plasmáticos asociadas al polimorfismo de la apolipoproteína E en un grupo de escolares de Quindío, Colombia. Biomédica. 2009:29:382-91.

17. Editorial. Estimating LDL ApoB: Infomania or Clinical Advance? Clin. Chem. 2008;54:5 782-784

18. Saghafi H, Mahmoodi MJ, Fakhrzadeh H, Heshmat R, Shafaee A, Larijani B. Cardiovascular risk factors in firstdegree relatives of patients with premature coronary artery disease. Acta Cardiol 2006; 61: 607-613.

19. Boekhold SM, Peters RJ, Fountoulaki K, Kastelein JJ, Sijbrand EJ. Molecular Variation at the apolipoproteína B gen locus in relation to lipids and cardiovascular disease: systenmatic metanalysis. Hum Genet 2003; 113:417-425

20. Guzman EC, Hirata MH, Quintao EC, Hirata RD. Association of apolipoproteín B gene polymorphism with cholesterol levels and response to fluvastatin in Brazilian individuals with risk for coronary heart disease. Clin Chem Lab Med 2000; 38:731-736

21. Zaman MM, Ikemoto S, Yoshiike N, Date CH, Yokoyama T, Tanaka H. Association of apolipoprotein genetic polymorphisms with plasma cholesterol in Japanese rural population. The Shibata Study. Arteriosclerosis Thrombosis and Vascular Biology. 1997;17:3495-3504

22. Corbo RM, Scacchi R, Mureddu L, Mulas G, Castrechini S, Rivasi AP. Apolipoprotein B, apolipoprotein E, and angiotensin converting enzyme polymorphisms in 2 Italian populations at different risk for coronary artery disease and comparison of allele frequencies among European populations. Human Biol. 1999; 71: 933-945

23. Puri RD, Tewari S, Sinha N, Ramesh V, Khan F, Singh VP, Agrawal S. Polymorphism in the apolipoproteína B-100 gene: association with plasma lipid concentration and coronary artery disease. Indian Heart J 2003; 55: 60-64

24. Ye $P$, Chen B, Wang S. Association of polymorphism of the apolipoproteína B gene with coronary heart disease in Han Chinese. Atherosclerosis 1995; 117:43-50

25. Aalto-Setala, KM. Tikkanen, M-R. Taskinen M, Nieminen P, Homberg, Kontula K. Xbal and C/G polymorphisms of the apolipoprotein B gene locus are associated with serum cholesterol and LDL-cholesterol levels in Finland. Atherosclerosis. 1988; 74: 47-54. 